Cinémas

Revue d'études cinématographiques

Journal of Film Studies

\title{
L'évolution de la fin : de La Jetée à 12 Monkeys
}

\section{Carolina Ferrer}

Volume 13, numéro 3, printemps 2003

Imaginaire de la fin

URI : https://id.erudit.org/iderudit/008707ar

DOI : https://doi.org/10.7202/008707ar

Aller au sommaire du numéro

Éditeur(s)

Cinémas

ISSN

1181-6945 (imprimé)

1705-6500 (numérique)

Découvrir la revue

Citer cet article

Ferrer, C. (2003). L'évolution de la fin : de La Jetée à 12 Monkeys. Cinémas, 13(3),

53-77. https://doi.org/10.7202/008707ar

\section{Résumé de l'article}

Plus de trente ans se sont écoulés entre la production de La Jetée (1962), de Chris Marker, et le film qu'elle a inspiré à Terry Gilliam, 12 Monkeys (1995). En comparant les deux productions, on peut observer que d'importants changements se sont opérés dans l'imaginaire contemporain : plutôt qu'une catastrophe nucléaire, c'est l'anéantissement provoqué par un fou solitaire qui menace le monde ; la participation des personnages féminins est devenue plus active ; la trame des événements dévoile une complexité croissante. Cependant, les voyageurs intertemporels des deux films expérimentent toujours un profond malaise devant une temporalité qui ne respecte pas les lois de la causalité. 


\section{L'évolution de la fin: de La Jetée à 12 Monkeys}

\section{Carolina Ferrer}

\section{RÉSUMÉ}

Plus de trente ans se sont écoulés entre la production de La Jetée (1962), de Chris Marker, et le film qu'elle a inspiré à Terry Gilliam, 12 Monkeys (1995). En comparant les deux productions, on peut observer que d'importants changements se sont opérés dans l'imaginaire contemporain: plutôt qu'une catastrophe nucléaire, c'est l'anéantissement provoqué par un fou solitaire qui menace le monde; la participation des personnages féminins est devenue plus active; la trame des événements dévoile une complexité croissante. Cependant, les voyageurs intertemporels des deux films expérimentent toujours un profond malaise devant une temporalité qui ne respecte pas les lois de la causalité.

\section{ABSTRACT}

More than thirty years have passed between the production of Chris Marker's La Jetée (1962) and the film it inspired, Terry Gilliam's 12 Monkeys (1995). By comparing the two films, one may observe some important changes in the contemporary imaginary: the mode of imagining the end of the world has been displaced from nuclear threat towards that of an annihilation provoked by a solitary madman; the participation of feminine characters becomes more active; the train of events reveals a growing complexity. However, the time-travellers of both films always experience a profound malaise before a temporality that does not respect the laws of causality. 
La Deuxième Guerre mondiale nous légua, outre les horreurs de la destruction et de ses millions de morts, deux icônes apocalyptiques incontournables: Auschwitz et Hiroshima. Dans les camps de concentration nazis, des milliers de personnes subirent les plus inimaginables atrocités, planifiées et exécutées avec une efficacité inébranlable. Pour mettre au point cette industrie de la mort, Hitler bénéficia non seulement de la participation de ses troupes, spécialement de l'élite des S.S., mais aussi de celle de nombreux scientifiques qui virent dans les camps de la mort un laboratoire où ils pourraient laisser libre cours aux plus noires expériences jamais tentées sur des humains.

En même temps, nazis et Alliés participaient à une course sur un autre front: le développement de la bombe atomique qui devait permettre à celui qui la posséderait le premier d'être le vainqueur de cette confrontation mondiale. Ainsi, cette autre association de militaires et de scientifiques généra, au sein du projet Manhattan, la première bombe nucléaire que les ÉtatsUnis n'hésitèrent pas à utiliser contre le Japon, dernière puissance de l'Axe encore au combat. L'anéantissement dont furent victimes les citoyens japonais laissa immédiatement présager que la guerre suivante, qui opposerait vraisemblablement les ÉtatsUnis et l'URSS, se jouerait d'abord dans les laboratoires où les savants s'acharnaient à perfectionner des armes toujours plus meurtrières, de destruction totale.

Nous ne devrions être aucunement surpris par l'existence d'alliances entre militaires et scientifiques dans l'imaginaire contemporain de la fin du monde. Au contraire, celles-ci semblent tout à fait naturelles dans les visions apocalyptiques nées de la guerre froide. Dans les représentations littéraires et cinématographiques de la fin du monde, même lorsque les populations sont exterminées directement ou indirectement à cause de l'essor de la science, la présence de scientifiques au sein des pouvoirs qui planifient l'avenir est constante. Tel est le cas dans 12 Monkeys, du réalisateur Terry Gilliam, ainsi que dans La Jetée, de Chris Marker, dont Gilliam s'est inspiré. Dans ces univers dévastés, l'un par un biologiste fou qui a répandu un virus mutant et l'autre par une guerre nucléaire, la possibilité de 
survie repose sur la capacité des savants de projeter des voyageurs dans le temps.

Les deux œuvres pourraient être résumées par la phrase d'ouverture de La Jetée: "Ceci est l'histoire d'un homme marqué par une image d'enfance." Cependant, dans 12 Monkeys, cette affirmation demeure un non-dit, que nous déduisons au fur et à mesure que le rêve obsessif du personnage principal, James Cole, est projeté en quatre versions différentes. L'homme en question, d'après Marker, est un prisonnier qui s'est battu au cours de la Troisième Guerre mondiale, à la suite de laquelle les survivants habitent des souterrains pour échapper à la radioactivité qui a presque éliminé la vie sur la planète. En revanche, le film de Gilliam met en scène non pas un héros de guerre mais un dangereux criminel qui, avec d'autres reclus, doit se soumettre "volontairement" à ceux qui détiennent le pouvoir, alors qu'un virus a anéanti la majeure partie de l'humanité et obligé les rares survivants à résister, cachés dans les souterrains urbains. Dans les deux films toutefois, le personnage est sélectionné pour voyager dans le temps et, ainsi, rapporter une solution qui permettra aux humains de rendre la Terre habitable; au cours de ses nombreux voyages, il tombe amoureux d'une femme appartenant au passé et, lors d'une de ses missions, il est tué dans un aéroport sous les yeux de celle-ci. C'est à ce moment que le spectateur comprend que la scène de sa mort et l'image d'enfance qui le hante évoquent le même événement, celui où, à huit ans, il a été témoin de son propre assassinat.

Malgré ces similarités entre les deux productions, nous proposons d'aborder ici trois aspects divergents fondamentaux. Premièrement, puisque plus de trente ans séparent les deux films, années au cours desquelles les tensions entre les blocs est-ouest se sont apaisées et où le mur de Berlin est tombé, la manière de penser la fin du monde, tributaire de ces contextes historiques, s'est obligatoirement transformée. Dans La Jetée, il s'agit d'une guerre nucléaire, alors que dans 12 Monkeys, un virus mutant élimine la quasi-totalité de l'humanité. Divergence qui se reflète aussi dans l'écart évident du format de production des films ${ }^{1}$.

Deuxièmement, le lien intimiste entre le voyageur et la femme du passé de la production de Marker est remplacé dans 
12 Monkeys par une relation entre une psychiatre et son patient qui finissent pas se rapprocher. Dans les deux cas cependant, l'histoire d'amour est vouée à l'échec puisqu'il faut que cette relation s'achève afin que le personnage puisse sauver l'humanité.

Troisièmement, la technologie employée pour envoyer les voyageurs dans le temps differe de façon considérable. Ainsi, dans le film de Gilliam, la multiplicité des voyages et les altérations qu'ils provoquent sur le cours des événements génèrent une dynamique plus complexe que celle de La Jetée.

\section{Modalité de la fin}

Au début des années 1990, N. Katherine Hayles définissait, dans Chaos Bound. Orderly Disorder in Contemporary Literature and Science, le postmodernisme culturel comme un processus de dénaturalisation de l'expérience humaine. Au cours du $\mathrm{XX}^{\mathrm{e}}$ siècle, explique-t-elle, il y a une prise de conscience à l'effet que l'expérience se compose de constructions sociales au lieu de découler seulement de faits naturels. La dénaturalisation se serait déroulée en quatre étapes: dénaturalisation du langage, du contexte, du temps et de l'être humain.

Évidemment, la dénaturalisation qui a lieu entre La Jetée et 12 Monkeys est celle du temps. Selon Hayles: "The rhythm of our century seemed predictable. World War I at the second decade; World War II at the fourth decade; World War III at the sixth decade, during which the world as we know it comes to an end" (Hayles 1990, p. 279).

Cette projection des conflits mondiaux est justement évoquée par Marker. Son scénario apocalyptique est clairement influencé par les icônes de la Deuxième Guerre mondiale que nous avons déjà mentionnées. D'une part, l'explosion de la bombe atomique ${ }^{2}$ signifie la destruction de Paris et il en résulte que «la plus grande partie du monde était inhabitable, pourrie par la radioactivité» (Marker). D'autre part, parmi les survivants, certains se considèrent vainqueurs tandis que d'autres, devenus leurs prisonniers, sont entassés dans les sous-sols de la ville. Les images montrent des lits superposés, tout à fait semblables à ceux des baraques nazies. Le choix de la Ville lumière comme première cible ne 
semble pas un hasard. Marker s'acharne à montrer la destruction de Paris, ville épargnée par les bombardements pendant la Deuxième Guerre mondiale et surtout maintenue presque intacte malgré l'ordre de Hitler de la faire incendier à la fin de l'Occupation. Ainsi, sa destruction montrerait le manque total d'intérêt pour préserver la culture. Par ailleurs, ceux qui échappent à l'anéantissement de la ville se réfugient dans les souterrains du palais de Chaillot, lieu chargé de signification historique et culturelle: d'une part, lors de la libération de Paris en 1944, le général Charles de Gaulle y prononça un discours pour saluer les combats des Alliés et tracer les grandes lignes de l'avenir de la France $^{3}$, d'autre part, ce bâtiment abrite de nombreux musées, notamment celui de l'Homme, et entre ses deux ailes s'étend l'esplanade des Droits de l'homme ${ }^{4}$.

Allant à l'encontre de l'humanisme que symbolise Chaillot, et agissant de la même façon que le docteur Mengele et ses collègues qui soumirent des milliers de déportés dans les camps de concentration à des expériences scientifiques dans des camps souterrains, un groupe de scientifiques pratique des expériences sur les prisonniers. Le résultat est que ces derniers périssent ou deviennent fous. Le but des recherches est de projeter un voyageur dans le temps qui rapporterait du futur des solutions pour rendre à nouveau viable la planète dévastée. Notons la pauvreté de l'équipement scientifique: un hamac, des bandeaux pour couvrir les yeux, des seringues sont les seuls instruments que possèdent les chercheurs.

Au-delà des difficultés physiques du périple intertemporel, les cobayes humains ne supportent pas d'habiter dans un autre temps. Après beaucoup d'échecs, l'homme marqué par une image de son enfance devient la cible des chercheurs. Les geôliers épient les rêves des détenus et découvrent l'obsession de cet homme pour un souvenir particulier. Après d'intenses sessions de piqûres, ils le préparent pour son voyage. Alors que, dans le présent, l'homme souffre intensément, dans le passé où il est projeté, il rencontre une femme qui accepte ses allées et venues, ses disparitions fréquentes, sans demander d'explications.

Dans 12 Monkeys, la situation est différente. Le film de Gilliam ne vise pas à représenter la réalité puisqu'il n'y a pas eu 
de Troisième Guerre mondiale dans les années 1990. À cet égard Hayles (1990, p. 280) remarque: "We cannot help suspecting that maybe it happened after all and we failed to notice. Consequently time splits into a false future in which we all live and a true future that by virtue of being true does not have us in it. » Le film de Gilliam se construit justement sur cette tension entre le véritable anéantissement de l'espèce humaine et une réalité alternative: dans ce cas précis, celle d'un fou qui croit voyager dans le temps.

Paul Watzlawick (1978) examine dans La Réalité de la réalité à quel point la constitution de la réalité se base sur les modes de transmission des messages. Parmi les multiples problèmes qu'il étudie, il mentionne celui de la possibilité de voyager à une vitesse proche de celle de la lumière. Depuis la théorie de la relativité d'Albert Einstein, nous savons que si un corps atteint une telle vitesse, il voyage dans le temps. Watzlawick imagine la possibilité d'un message tachyonique ${ }^{5}$ en remarquant que cet événement signifierait que les réponses arriveraient avant les questions, et qu'en conséquence, le message expédié serait tout à fait déconcertant pour le récepteur. Ce qui est le cas du message de Cole en 1990, au point qu'on diagnostique chez lui une maladie mentale.

Par ailleurs, la modalité de fin du monde mise en scène dans 12 Monkeys ne correspond pas à un scénario de confrontation armée, mais à celui où un scientifique travaille auprès d'un prix Nobel en médecine à l'élaboration d'un virus mutant. Ce scénario concorde avec le contexte de 1990 où l'empire soviétique s'étant effondré, la guerre froide vient de se terminer. Puisque l'épidémie n'atteint pas les animaux, sa propagation est rapidement endossée par un groupe écologiste, l'Armée des douze singes. De cette façon, dans le film de Gilliam, nous voyons clairement que la hantise de la bombe atomique mise en image par Marker, est remplacée ici par la crainte qu'un homme seul, participant aux recherches les plus avancées en biochimie, puisse déraper et vouloir en finir avec la vie humaine ${ }^{6}$.

D'un film à l'autre, nous observons aussi un déplacement géographique: Gilliam situe le commencement de la fin en Amérique, territoire jamais atteint pendant la Deuxième Guerre 
mondiale. L'épidémie est d'ailleurs déclenchée à Philadelphie, lieu chargé de signification puisque la Déclaration d'indépendance des États-Unis fut signée dans cette ville. La Cloche de la liberté qui, en 1776, annonça la première lecture publique de cette déclaration, porte une inscription biblique: "Proclaim liberty throughout all the land unto all inhabitants thereof ${ }^{7}$." Dans cette vision cinématographique, Philadelphie ${ }^{8}$ devient le point de départ de la maladie qui se répand dans le reste du monde: San Francisco, New Orleans, Rio de Janeiro, Rome, Kinshasa, Karachi, Bangkok, Beijing, jusqu'à couvrir la planète.

Après l'hécatombe, le monde du futur est dominé par les scientifiques. Ceux-ci sélectionnent un prisonnier, James Cole, pour une expédition à la surface de la Terre, au cours de laquelle il élabore une thèse voulant que l'Armée des douze singes ait répandu le virus. Grâce à sa mémoire et à sa capacité d'observation, il est choisi pour voyager dans le temps et rapporter des renseignements sur le virus avant qu'il ne subisse des mutations. Cependant, Cole arrive à destination avant le temps programmé et son discours sur la fin du monde, énoncé de manière violente, provoque son internement dans un hôpital psychiatrique. La médecin chargée de son cas, la docteure Kathryn Railly, jouera un rôle fondamental dans l'histoire. Se trouvant absolument dépourvu de repères chronologiques, Cole n'arrive pas à convaincre les médecins que la majorité des humains périra, pas plus qu'il ne parvient à communiquer avec les scientifiques du futur. Pendant sa réclusion, il fait la connaissance de Jeffrey Goines, fils du prix Nobel en médecine, qui par la suite formera l'Armée des douze singes. Au cours de ce voyage dans le futur, tous les éléments sont mis en place pour aboutir à l'assassinat de Cole, qui ne pourra échapper à son destin.

À partir de l'observation des recherches des scientifiques de ce monde souterrain, nous pouvons déduire la quantité d'expériences accomplies, indice important pour l'étude de la dynamique du film: les prisonniers sont enfermés dans des cages, comme jadis les singes dans le laboratoire du docteur Goines; un code-barres tatoué dans le cou donne directement accès au dossier du détenu; dans la salle d'interrogatoires, les scientifiques sont assis autour d'une table, disposition semblable à celle 
des psychiatres de 1990; la victime est suspendue dans les airs, assise sur un siège selon un schéma inspiré - de très près - par l'œuvre de Lebbeus Woods, Neomechanical Tower (Upper) Chamber'. Là-haut, le prisonnier n'est qu'un réceptacle d'informations. Premièrement, on lui donne des renseignements provenant du passé afin qu'il puisse accomplir sa tâche, ensuite on l'interroge pour savoir ce qu'il a appris lors de son expédition. Toutes ces installations indiquent sur le plan sémiotique une présence infiniment plus importante de la science dans 12 Monkeys que dans La Jetée.

En revanche, on ne peut s'empêcher de voir une correspondance entre la conception formelle des deux films et les modalités de fin du monde qui s'y trouvent reflétées. Les images fixes dans l'œuvre de Marker riment parfaitement avec la fixation d'un point dans le temps: l'instant qui sépare l'avant et l'après de l'utilisation des armes nucléaires. En 1945, les bombardements d'Hiroshima et de Nagasaki par le gouvernement des États-Unis associèrent pour toujours armes nucléaires et apocalypse. Nous pouvons même affirmer que cette révélation est annoncée par une rupture dans l'histoire des découvertes scientifiques. En effet, la fabrication de la première bombe atomique fut le résultat d'une longue histoire de coopération internationale entre de nombreux scientifiques qui échangèrent librement leurs découvertes pendant la première moitié du $\mathrm{XX}^{\mathrm{e}}$ siècle dans le but de dévoiler le mystère de l'atome et de son noyau ${ }^{10}$. Cependant, la découverte de la fission nucléaire ébranla l'esprit pacifiste de maints scientifiques et, dès 1938, les savants eurent la certitude que les connaissances acquises se traduiraient par un changement tragique du cours de l'histoire de l'humanité. Le photo-montage de La Jetée évoque l'immobilité presque totale à laquelle serait condamnée la planète lors d'une guerre nucléaire. Le seul recours des survivants dans l'univers de Marker est de récupérer des souvenirs, encore des images fixes, du temps d'avant la guerre.

Le film de Gilliam, contrairement à celui de Marker, semble suivre l'évolution du virus qui mute constamment afin de se rendre indestructible. Mouvement qui accompagne la propagation de l'épidémie, décrit par la fonction logistique ${ }^{11}$. Cette 
structure ramifiée, chaotique, n'est pas constante dans 12 Monkeys. Par exemple, nous voyons comment les animaux libérés par l'Armée des douze singes, qui déclenchent d'incroyables embouteillages, contrastent ouvertement avec ceux que le couple du film de Marker apprécie dans le Muséum national d'histoire naturelle.

Ce genre de trajectoire est observable aussi lorsque, au lieu de rester ancré sur une seule image de son enfance, le rêve de Cole se modifie en fonction des événements. Au début, l'homme qui court avec une valise est impossible à identifier. Quand il fait la connaissance de Jeffrey, il le voit dans son évocation. Par la suite, Cole "ajuste" l'image du rêve: l'homme devient l'assistant du laboratoire, vision qui correspond à la réalité. Par un processus de rétroalimentation, ces altérations se traduisent en actions qui, à leur tour, bouleversent le cours des événements.

Les deux films présentent une forte correspondance entre la modalité de la fin du monde et le format de production cinématographique, contrastant avec les nombreuses différences entre les scénarios. Par ailleurs, les mondes intimes proposés respectivement par Marker et Gilliam se distinguent significativement.

\section{Intimité}

Dans Le Prochain Amour (1996), de l'écrivain français Yves Simon, roman qui tisse de nombreux liens avec les films de Marker et de Gilliam qui nous intéressent ici, le narrateur raconte ses peines d'amour avec Irène, une hôtesse de l'air. Les visions du monde des amants sont trop différentes l'une de l'autre pour que leur amour soit possible. Alors que le narrateur fait tout pour Irène, celle-ci disparaît pendant des jours, le trompe à plusieurs reprises et, au bout du compte, affiche un comportement tout à fait incohérent. Ainsi, le "prochain amour» meurt malgré la certitude que le narrateur partage avec cette femme qu'ils se sont mutuellement rêvés avant leur première rencontre.

Les rêves, l'amour impossible, les images d'aéroports et les différences d'espace-temps provoquées par de nombreux décalages horaires ne sont pas les seuls points communs entre le livre de Simon, La Jetée et 12 Monkeys. Le film de Marker apparaît comme l'intertexte du roman lorsque le narrateur raconte 
l'histoire d'amour de son ami, Anton Vladimir. Plusieurs années après leur séparation, Roberta entre en contact avec Anton, son ancien amant, pour lui apprendre qu'elle a un cancer. Elle lui dit aussi qu'elle désire passer ses derniers jours auprès de lui, que son visage soit le dernier qu'il lui sera donné de voir. Leur histoire remonte aux années qui suivirent la Deuxième Guerre mondiale. Roberta avait été marquée par leur première nuit d'amour; Vladimir lui avait dit vouloir faire du cinéma et il l'avait troublée par l'idée de son premier film, présenté ainsi :

\begin{abstract}
C'est l'histoire d'un homme marqué par une image d'enfance. La scène qui le troubla par sa violence, et dont il ne devait comprendre que beaucoup plus tard la signification, eut lieu sur la grande jetée d'Orly, quelques années plus tard avant le début de la Troisième Guerre mondiale (Simon 1996, p. 192).
\end{abstract}

Se sachant sur le point de mourir, Roberta appelle Vladimir. Ils sont toujours amoureux et se marient. Mais le destin leur assène un nouveau coup dur: Vladimir meurt.

Lallusion est intéressante. Tout comme dans les films de Marker et de Gilliam, malgré la mort qui traque les femmes, imminente, ce sont les hommes qui meurent les premiers. Toutes ces histoires d'amour sont fauchées malgré le destin qui persiste à lancer les amants l'un vers l'autre.

Dans le film de Marker, l'homme qui est marqué par une image d'enfance est envoyé dans le passé où il rencontre une femme. Bien qu'il disparaisse et réapparaisse à maintes reprises, elle accepte cette étrange relation et appelle cet homme "son Spectre». Les amants sont "sans souvenirs, sans projets", ces derniers étant impossibles, car l'homme possède la certitude que son amante mourra avec la majorité des habitants de la planète, qui sera bientôt anéantie. Il sait aussi que lorsque les scientifiques obtiendront de lui des ressources en provenance du futur pour sauver l'humanité, ils vont le sacrifier ${ }^{12}$. Cependant, les êtres de l'avenir l'invitent à vivre dans leur temps. Au lieu d'accepter leur proposition, il demande à revenir dans le passé où cette femme l'attend. De retour à l'aéroport, il s'élance à sa rencontre. Marker consacre plusieurs plans à cette course. Après tout ce qu'il a vécu, 
la guerre, l'emprisonnement, les expériences scientifiques, les voyages dans le temps, l'homme n'a qu'un désir: être avec cette femme. Il pense un instant que lui-même enfant doit se trouver à l'aéroport, mais cette idée ne le détourne pas de son élan.

Il court vers elle au bord de la jetée. À ce moment, un homme du camp, qui l'avait suivi, tire sur lui. À l'instant de sa mort, il se rend compte que cette scène est celle l'ayant marqué dans son enfance: à huit ans, il a vu sa propre mort et «il comprit qu'on ne s'évadait pas du Temps».

Comparons maintenant cette image de La Jetée avec son équivalent dans 12 Monkeys. Pour commencer, dans le film de Gilliam, la mort de James Cole n'est pas la dernière image du film. Elle est suivie du départ de l'enfant de l'aéroport avec ses parents et de la rencontre entre l'assistant du docteur Goines et la scientifique du futur dans l'avion. Nous reviendrons sur cette scène. Pour l'instant, regardons de plus près le moment où Cole est atteint par les tirs.

James reçoit l'ordre de tuer un homme. La docteure Railly, sa psychiatre et la femme qu'il aime, lui indique qui est celui qu'il doit tuer. La course de Cole ne le conduit pas vers la femme qu'il aime, comme dans La Jetée, mais plutôt vers l'assistant du docteur Goines. Railly court derrière lui, essaye d'empêcher les policiers de tirer sur James et le prend dans ses bras au moment de sa mort. Par ailleurs, la scène, filmée en caméra lente, s'arrête sur le visage de la femme qui cherche du regard James enfant puisqu'elle sait qu'il se trouve là. Son regard croise celui de Cole à huit ans qui semble troublé et restera hanté par ce souvenir. Dans la tristesse du regard de la femme, réside la vérité de tout ce qui suivra par rapport à cet enfant, à son amour, à l'humanité. Cette image différencie fondamentalement les deux films. Dans l'œuvre de Marker, le voyageur intertemporel a épargné à la femme qu'il aime la souffrance qui l'attend en lui masquant l'avenir. Dans celle de Gilliam, non seulement la docteure Railly est au courant de ce qui viendra, mais elle tente d'aider Cole. Cependant, elle ne peut pas lui venir en aide puisque tout le monde croit que sa raison a basculé, qu'elle est la victime de la folie de Cole. Kathryn Railly semble atteinte de l'objet de ses études: le syndrome de Cassandre. 
Figure mythique de l'Antiquité, Cassandre est la déesse qui prévoit le futur, mais comme personne ne la croit, ses prédictions sont impuissantes à empêcher les malheurs. Le nom de Cassandre est donc lié depuis l'origine à la destruction, comme le rappelle Goudot (1999, p. 8): "On pourrait lire la guerre de Troie [...] en suivant le déroulement de ses révélations.» Par ailleurs, cette guerre correspond à une image fondatrice de l'anéantissement dans la culture gréco-romaine: "Premier génocide, injuste guerre d'expansion coloniale, autodestruction d'une ville parmi les plus opulentes, ou bien encore guerre fratricide, comme on voudra la considérer: elle ne cesse depuis des siècles de hanter l'imaginaire occidental.»

L'image de Cassandre est reprise dans 12 Monkeys à travers la figure de la psychiatre. Après avoir fait la connaissance de Cole, celle-ci entreprend une recherche sur l'hallucination mentale particulière qui consiste à se croire en possession de renseignements sur la fin du monde. Elle étudie alors un grand nombre de personnages au long de l'histoire qui furent atteints de cette maladie qu'elle nomme le «syndrome de Cassandre». Parmi ces cas étudiés, elle mentionne celui d'un homme qui était sur la ligne de front pendant la Première Guerre mondiale. En voyant la photo de cet homme, on se rend compte qu'il s'agit de José, un camarade de prison de James. Cette photographie est un élément clé de la narration puisque Cole y apparaîtra soudainement lorsque, à cause d'une erreur de la machine à voyager dans le temps, il fait irruption par accident en 1917 aux côtés de José. Cole est alors blessé par une balle à la jambe. Puisqu'il repart immédiatement vers l'année 1996, c'est Railly qui extrait la balle de sa jambe. Ainsi, ce projectile, lié à d'autres renseignements que Cole fournit à Railly sur son futur proche et que la psychiatre parvient très vite à vérifier, constitue une pièce à conviction qui achève de persuader la psychiatre de l'histoire initialement racontée par Cole sur les voyages dans le temps. Désormais, Kathryn Railly commence elle aussi à annoncer la mort de quatre-vingt-dix neuf pour cent de la population mondiale.

Rapprochons maintenant la scène de la mort de Cole et l'histoire de Cassandre racontée par la romancière allemande, 
Christa Wolf. Cette réécriture du mythe antique est intéressante à cause de la source ayant inspiré la création de Wolf. Tandis que, dans les années 1990, de part et d'autre du rideau de fer, des missiles nucléaires pouvaient éliminer la vie sur la planète, elle s'interroge: «La question que je me suis posée en commençant à m’intéresser au thème de Cassandre [...] était la suivante : quand et comment cette tendance à l'autodestruction est-elle apparue dans la pensée et dans l'action occidentales?» (Wolf 1999, p. 130). Wolf remonte dans le temps et recule jusqu'à ce qu'elle appelle la "protohistoire». Elle s'interroge alors : "Comment se fait-il qu'un dieu [Apollon], mâle, fasse le don de la voyance à une femme alors que, dans les temps très anciens, il n'y avait que des déesses, des prêtresses ou des voyantes?» (Wolf 1999, p. 132). L'écrivaine prend conscience des changements qui se sont produits au cours de l'histoire concernant la place des femmes dans la société: "Les déesses de la terre et de la fécondité, qui imposaient le respect, furent évincées au profit de dieux mâles et de cette pléiade de dieux que fut l'Olympe des Grecs» (Wolf, 1999, p. 133). En suivant Wolf, nous pouvons voir comment les femmes ont été poussées en marge de l'histoire, reléguées à un rôle passif, comme le personnage féminin de La Jetée.

Par contre, dans 12 Monkeys, la docteure Railly joue un rôle dans l'action qui se dessine, son personnage se superposant à celui de la mythique Cassandre. Au début du film, elle est appelée par la police pour évaluer la santé mentale de cet homme qui divague et parle de l'apocalypse à venir. Elle décide de le faire interner, puis établit un lien de confiance avec Cole, d'abord en prenant sa défense auprès de ses collègues, puis auprès de la police, pour finalement endosser et même propager son discours apocalyptique.

L'histoire d'amour entre Railly et Cole prend une place moins importante que celle qui se déroule dans La Jetée. Elle surgit vers la fin du film, lors du dernier voyage de Cole. La psychiatre est maintenant convaincue de ce que raconte James, alors que ce dernier veut au contraire qu'elle le guérisse de ce qu'il voit comme une maladie, cette autre réalité qu'il aurait créée: s'il est fou, l'humanité est sauve, alors que s'il dit vrai, l'humanité est 
condamnée. Railly est profondément émue par le désir de Cole de voir la planète épargnée du virus. Elle devient son guide dans cette réalité inconnue et veut lui offrir quelques jours au bord de la mer, car elle est convaincue que tout finira bientôt. Elle espère qu'ils passeront le temps qui leur reste dans un petit paradis, mais ils n'arriveront jamais à voir la mer. Sans doute pour accentuer le fait que leur relation est vouée au désastre, Gilliam a choisi le tango comme bande sonore du film. Les paroles de ce genre musical racontent toujours des tragédies insurmontables pendant que la sensualité des pas de danse montre l'attraction fatale qui réunit le couple.

La différence entre le film de Marker et celui de Gilliam quant aux rôles des personnages féminins reflète en quelque sorte les importants changements survenus dans le rôle des femmes, dans la société occidentale, depuis les années 1960. Empruntons les dernières paroles prononcées par Cassandre dans le roman de Wolf pour illustrer dans quelle mesure elles pourraient correspondre aux idées de Railly pendant la mort de Cole:

Bien-aimé. Tu n'as pas dit que cela ne t'arriverait pas. $\mathrm{Ou}$ bien que je pourrais t'en préserver. Contre une époque qui a besoin de héros, nous ne pouvons rien faire, tu le savais aussi bien que moi. Tu as jeté dans la mer l'anneau en serpent. Je devrai aller loin, très loin, as-tu dit, et sans savoir ce qui m'attend.

Moi, je reste.

Que la douleur nous fasse souvenir l'un de l'autre. C'est à elle que nous nous reconnaîtrons plus tard, quand nous nous retrouverons, si plus tard il y a.

La lumière s'éteignit. S'éteint.

Ils arrivent (Wolf 1985, p. 128).

On pourrait croire ces mots prononcés par Railly, lorsque la caméra offre un gros plan de son visage à la fin du film, sans que l'on sache s'ils sont adressés à Cole enfant ou à Cole qui agonise dans ses bras. D'ailleurs, la dernière phrase est textuellement prononcée par Cole à la fin de son deuxième voyage dans le passé. Marquée par sa rencontre avec Cole, Railly est devenue pareille aux fous qu'elle-même étudiait. De plus, sa faculté de prophétesse est reconnue par un autre de ses patients, Jeffrey 
Goines $^{13}$ : "She knew I was gonna lead the Army of the Twelve Monkeys into the pages of history before it ever even occurred to me. She knows everything I'm ever gonna do before I know it myself. " Bien qu'il se trompe à propos du moyen par lequel Railly est informée sur les événements futurs, Jeffrey lui accorde néanmoins qu'elle puisse prédire le futur.

Jadis, elle aurait classé son propre cas comme une manifestation du syndrome de Cassandre. Tel est pris qui croyait prendre: la voilà suspectée d'avoir la maladie dont elle est la spécialiste. C'est une des multiples boucles qui se dessinent dans ce film présentant une dynamique très particulière à cause des voyages dans le temps.

\section{Dynamique}

Le physicien Paul Davies (2002, p. 50) propose la construction d'une machine à explorer le temps en "trois étapes non faciles ", dans un numéro spécial de Scientific American, consacré au thème du temps. Au-delà des problèmes liés à l'élaboration d'une telle machine, il parle des paradoxes qui surgiraient si les voyages dans le temps devenaient possibles. Si un passager temporel essayait de changer le passé, nous pourrions être confrontés à des situations impossibles comme, par exemple, s'il tue sa mère avant sa propre naissance. Cependant, le voyageur peut faire partie du passé dans la mesure où la consistance causale se maintient.

En plus des paradoxes qu'ils engendrent, les voyages dans le temps nous confrontent au problème des flèches du temps. Dans Une brève histoire du temps, le physicien Stephen W. Hawking (1989, p. 187) indique:

Il y a au moins trois flèches du temps différentes. D'abord, il y a la «flèche thermodynamique " du temps, la direction du temps dans laquelle le désordre ou l'entropie croît. Ensuite il y a la «flèche psychologique». C'est la direction selon laquelle nous sentons le temps passer, dans laquelle nous nous souvenons du passé mais pas du futur. Enfin, il y a la «flèche cosmologique", direction du temps dans laquelle l'univers se dilate au lieu de se contracter. 
Ces trois flèches, nous explique Hawking, pointent dans la même direction. Observons ce qui arrive dans les deux films par rapport aux paradoxes et aux flèches du temps.

Les scientifiques de La Jetée administrent de mystérieuses piqûres aux cobayes humains pour les expédier ailleurs dans le temps. Il n'y a aucune explication sur le fonctionnement de ce mécanisme. Seul le résultat est connu: c'est une catastrophe. Les hommes meurent ou deviennent fous parce qu'ils perdent tous leurs repères en surgissant dans un autre monde. Les scientifiques trouvent alors un homme qui revient continuellement sur un souvenir d'enfance. Puisqu'il est capable d'habiter cette image, peut-être arrivera-t-il à survivre dans le passé aussi bien que dans le futur. Au début de l'expérience, il s'agit de lui faire retrouver des représentations mentales, des souvenirs du temps de paix. Ensuite, il rejoint le passé et reprend contact avec le monde d'avant la destruction où il ne rencontre qu'une femme à plusieurs reprises. À travers ces périples, sa présence dans le passé ne semble pas altérer l'histoire.

Ensuite, l'homme est envoyé dans le futur. Il n'arrive pas à se situer dans ce monde où il ne possède pas de repères. Les humains du futur ont une apparence différente: sur leur visage, ils ont une étrange masse adipeuse qui rappelle curieusement une prise électrique. Après qu'il ait obtenu leur aide, les habitants du futur communiquent avec lui pour lui offrir de les rejoindre. Ces personnes sont légèrement différentes de celles rencontrées lors de son premier voyage dans le futur: certaines ont deux de ces espèces de prises électriques sur le front au lieu d'une. Apparemment, l'aide qu'eux-mêmes ont fourni à ce monde du passé a altéré leur propre existence.

La difficulté des prisonniers à supporter les voyages dans le temps est due à l'écart qu'ils ressentent entre la flèche psychologique et les deux autres flèches, même si ce qu'ils perçoivent n'est que la divergence entre la flèche psychologique et la flèche thermodynamique. Mais ce n'est pas une mince affaire que de se déplacer dans l'espace-temps puisque, comme l'affirme André Comte-Sponville: "Être, c'est être présent en un point de l'espace-temps.» (1996, p. 265) Et les voyageurs se trouvent soudainement dépourvus de tout repère. Comment cet homme 
en particulier arrive-t-il alors à surmonter l'épreuve? Grâce à sa faculté de revenir plusieurs fois sur une même vision. Cependant, c'est à cause de cette même force spirituelle qu'il est condamné puisqu'il veut s'installer dans un espace-temps particulier, celui où la femme l'attend. Dans La Jetée, on ne cesse de nous répéter que le couple se trouve sans passé et sans avenir. Mais lors de leur dernière rencontre, s'ils se sont apprivoisés, c'est justement parce qu'ils ont accumulé des expériences ensemble. Ils possèdent donc un passé en tant que couple, même si ce passé est fragmenté par les disparitions inexplicables de l'homme. À ce moment, le besoin d'un avenir devient aussi évident et c'est ce que l'homme demande, mais c'est plutôt la mort qui l'attend. Sans sa mort, le reste de l'histoire aurait été impossible puisque l'image qui l'a marqué dans son enfance est celle de son assassinat. La consistance causale des événements est satisfaite.

La situation de 12 Monkeys est semblable du point de vue du couple, mais le rapport à l'humanité differe. Pour apprécier ces différences, nous devons d'abord analyser la dynamique des voyages dans le temps ainsi que leur effet sur le cours de l'histoire. Puisque au long du film, notre point de vue accompagne celui de Cole, concentrons-nous sur les voyages de ce dernier. La figure 1 (page 70) représente un schéma des voyages de ce personnage et de ceux qui sont immédiatement liés à son histoire. Les lignes noires épaisses correspondent au cours du temps : en principe, une ligne unique. Cependant, comme l'explique Watzlawick (1978, p. 225):

[C]haque fois qu'un voyageur du temps pénètre dans le passé, l'univers se sépare en deux bandes temporelles. L'une d'elles est la continuation de l'état antérieur des choses, tandis que l'autre est le début d'une réalité entièrement nouvelle où l'histoire peut suivre un cours entièrement différent.

Ainsi, chacun des voyages de Cole vers le passé, indiqués par les lignes noires minces, provoque une bifurcation de sorte que, au moment de retourner vers le futur, parcours indiqué par les lignes noires en pointillé, il débouche non pas sur la ligne temporelle d'où il est initialement parti, mais sur une nouvelle ligne temporelle. 

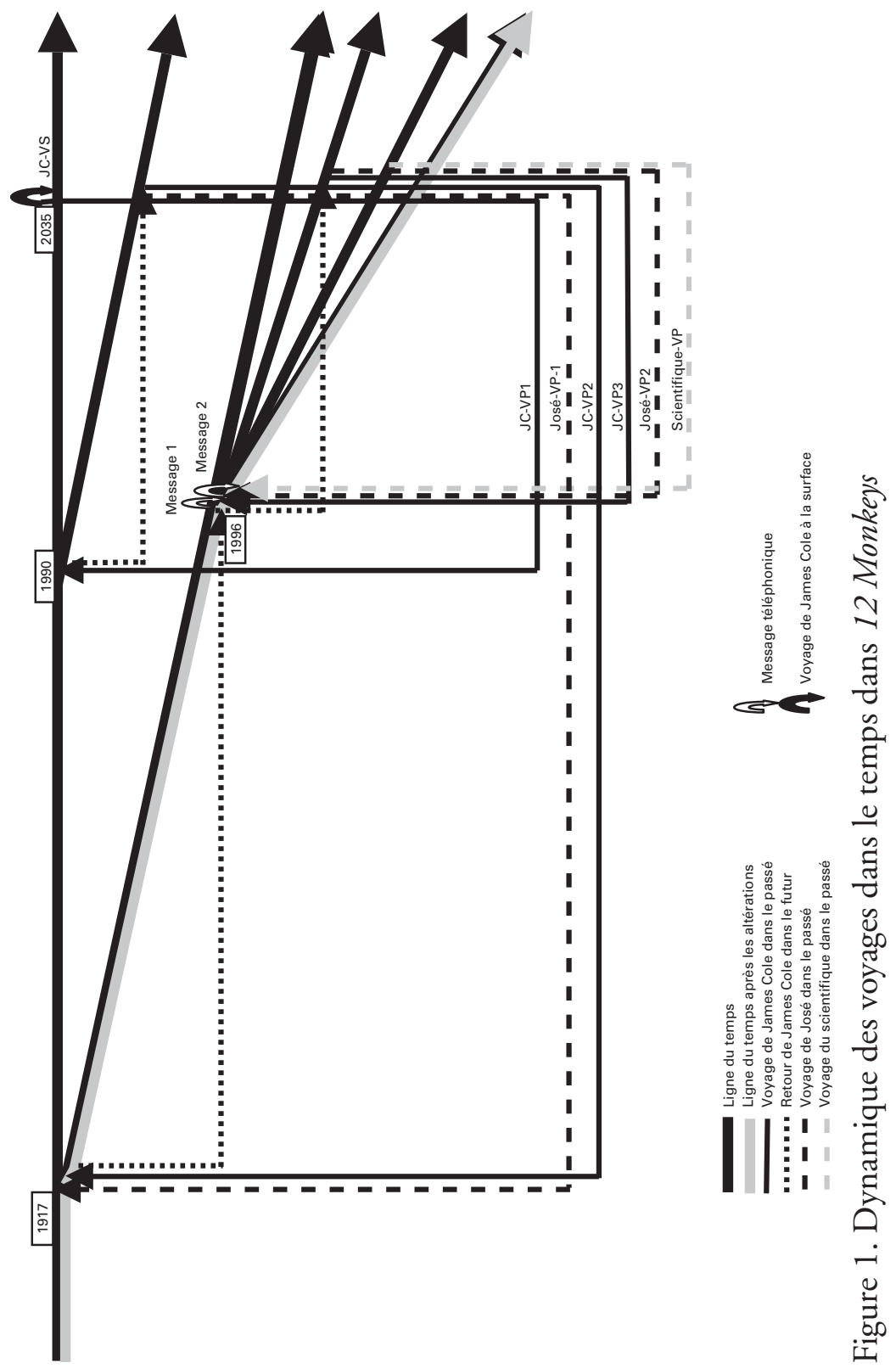
Le premier voyage de Cole se fait vers la surface, indiqué par une flèche noire qui se retourne sur elle-même. C'est au cours de ce premier voyage qu'il élabore son hypothèse selon laquelle l'Armée des douze singes a propagé l'épidémie. La ligne du temps n'en est pas pour autant altérée.

Le premier voyage de Cole dans le passé devait ramener celuici à l'an 1996 mais, à cause d'une erreur, il est ramené à l'an 1990, ce qui provoquera les résultats inattendus que nous avons déjà examinés. De retour à l'an 2035, Cole rejoint un monde modifié par rapport à celui de son départ.

Lors du deuxième voyage de Cole dans le passé, l'univers se trouve doublement changé: d'abord à cause de sa visite à l'an 1990 et ensuite par son détour accidentel par l'an 1917. En fait, cette visite inespérée à l'an 1917 a même altéré l'univers de 1990, visité par Cole lors de son voyage précédent, d'où l'impression étrange de Railly qui semble se souvenir de lui. C'est qu'elle l'a vu sur la photo de 1917 , même si celle-ci n'a pas encore été prise lors de son voyage de 1990 !

L'explication de ce phénomène se trouve dans le fait que chaque fois que l'univers se sépare en deux bandes, une seule d'entre elles aboutit à un résultat concret alors que l'autre correspond à l'état des choses telles qu'elles auraient été si la séparation n'avait pas eu lieu. Elle n'est donc plus «fonctionnelle». Ainsi, le cours du temps «réel» est celui indiqué par la ligne grise épaisse qui incorpore toutes les visites intertemporelles.

Nous voici face à la véritable complexité du monde de Gilliam, qui ne tient pas seulement compte du voyageur intertemporel Cole, mais des multiples autres voyageurs dans le temps. Sur la figure 1, nous trouvons seulement représentées les deux excursions de José et celle du scientifique; cependant il y en a bien d'autres, notamment celle du gardien de l'hôpital psychiatrique, celle du mendiant, celle du prédicateur. Ainsi, la ligne du temps de 12 Monkeys comporte la même quantité de points d'inflexion que de visites intertemporelles.

Par ailleurs, la fréquence des voyages intertemporels augmente une fois qu'est mis en place le mécanisme consistant à enregistrer des messages dans la boîte vocale des nettoyeurs de tapis, phénomène représenté par des flèches blanches qui se 
retournent sur elles-mêmes. Grâce à la reconstitution de cette bande de messages, les scientifiques de l'année 2035 profitent d'un système de rétroalimentation rapide et efficace. Du coup, il n'est plus nécessaire de faire revenir Cole du passé pour qu'il les informe sur ses recherches. Le système est même utilisé par Railly pendant le dernier voyage de Cole. Elle téléphone et laisse un message que les scientifiques du futur ont reconstitué et qu'ils ont fait entendre à Cole avant son départ. Le futur de 2035 incluait déjà ce que la psychiatre n'avait pas encore fait en 1996.

Juste avant son départ pour la mer, Cole, voulant se libérer du futur, enregistre dans la boîte vocale que l'Armée des douze singes a libéré les animaux du jardin zoologique, mais n'a pas propagé le virus. Les scientifiques du futur réagissent en envoyant José qui donne une arme à Cole et lui indique un homme à liquider. Ils savent déjà que la docteure Railly lui dira que l'homme à abattre est l'assistant du docteur Goines. Avec la police à ses trousses, Cole n'atteint pas son objectif et les détectives le tuent. Cole enfant en est témoin et la causalité est parfaitement comblée.

Mais l'histoire ne s'arrête pas là. Les scientifiques sont informés, possiblement par José, de l'échec de Cole, même si nous ne le voyons pas dans le film. Ce dont nous sommes bel et bien témoins, c'est de la rencontre entre l'assistant du docteur Goines et la femme scientifique du futur. Après avoir réussi à échapper à Cole, lorsque l'assistant entre dans l'avion avec sa valise contenant le virus à l'état pur, nous reconnaissons sa voisine de siège: c'est la seule femme savante qui participait aux interrogatoires de Cole en $2035^{14}$. À ce moment, nous comprenons que les habitants du futur sont sur le point de réussir: ils parviendront à capturer des échantillons du germe avant sa mutation et pourront donc freiner l'anéantissement de l'humanité.

\section{La fin de la fin}

Nous pouvons pousser plus loin cette capacité de correction du passé et imaginer des voyageurs qui parviendraient à arrêter l'assistant du docteur Goines avant qu'il ne débouche les tubes dans lesquels se trouve le virus, pendant l'inspection à l'aéro- 
port. Si nous considérons cette alternative, il n'y pas de fin du monde et la seule trace des "événements» sont les divagations d'un malade mental. Rappelons-nous le début du film: sur un écran apparaît une note avec les déclarations d'un patient schizophrénique qui annonce que quatre-vingt-dix neuf pour cent de l'humanité périra victime d'un virus. Nous pouvons y voir la réalité ultime proposée par Gilliam.

Remarquons que notre perception des faits est colorée par le point de vue de Cole, que nous suivons tout au long de l'histoire. Cependant, pour un observateur distrait, la ligne du temps est celle indiquée en gris et les lignes alternatives n'existent pas, tel que nous le voyons dans la figure 2 (page 74), qui représente la perception du temps. D'ailleurs, pour cet observateur, la "réalité " est d'autant plus simple qu'il ne perçoit même pas les inflexions causées par les voyages intertemporels. C'està-dire que le cours du temps est perçu comme une ligne droite similaire à la première représentation dans la figure 1 . Un tel observateur ne soupçonne pas que sa linéarité cache l'action des habitants du futur, et nommément ici, le sacrifice de Cole. Revenons à ce dernier, qui est étendu sur le sol de l'aéroport dans les bras de Railly, remarquant pour l'éternité, cet enfant qui est lui-même.

Si tout semble modifiable, même la fin du monde, il y a un fait qui demeure immuable: la mort de Cole. Pour que l'humanité soit épargnée, il doit être marqué par son souvenir d'enfance, il doit mourir, donc l'amour de Cole et de Railly est sans issue. De ce point de vue, le futur du couple ne se différencie pas de celui des amants de La Jetée.

Dans 12 Monkeys, même la modalité de la fin est changeante: la guerre atomique, immuable et mondiale de La Jetée, a été remplacée par un virus mutant qu'un homme seul déplace d'un pays à l'autre. Il s'agit d'une version apocalyptique «subtile» qui offre beaucoup de possibilités de manœuvres aux agents qui participent à l'opération de sauvetage de l'humanité. Avec un groupe de voyageurs intertemporels et la mise au point d'un système de messagerie, les habitants du futur pourraient même parvenir à empêcher la propagation du virus. Il suffit de trouver un bouc émissaire qui se promène à ses risques et périls dans le 


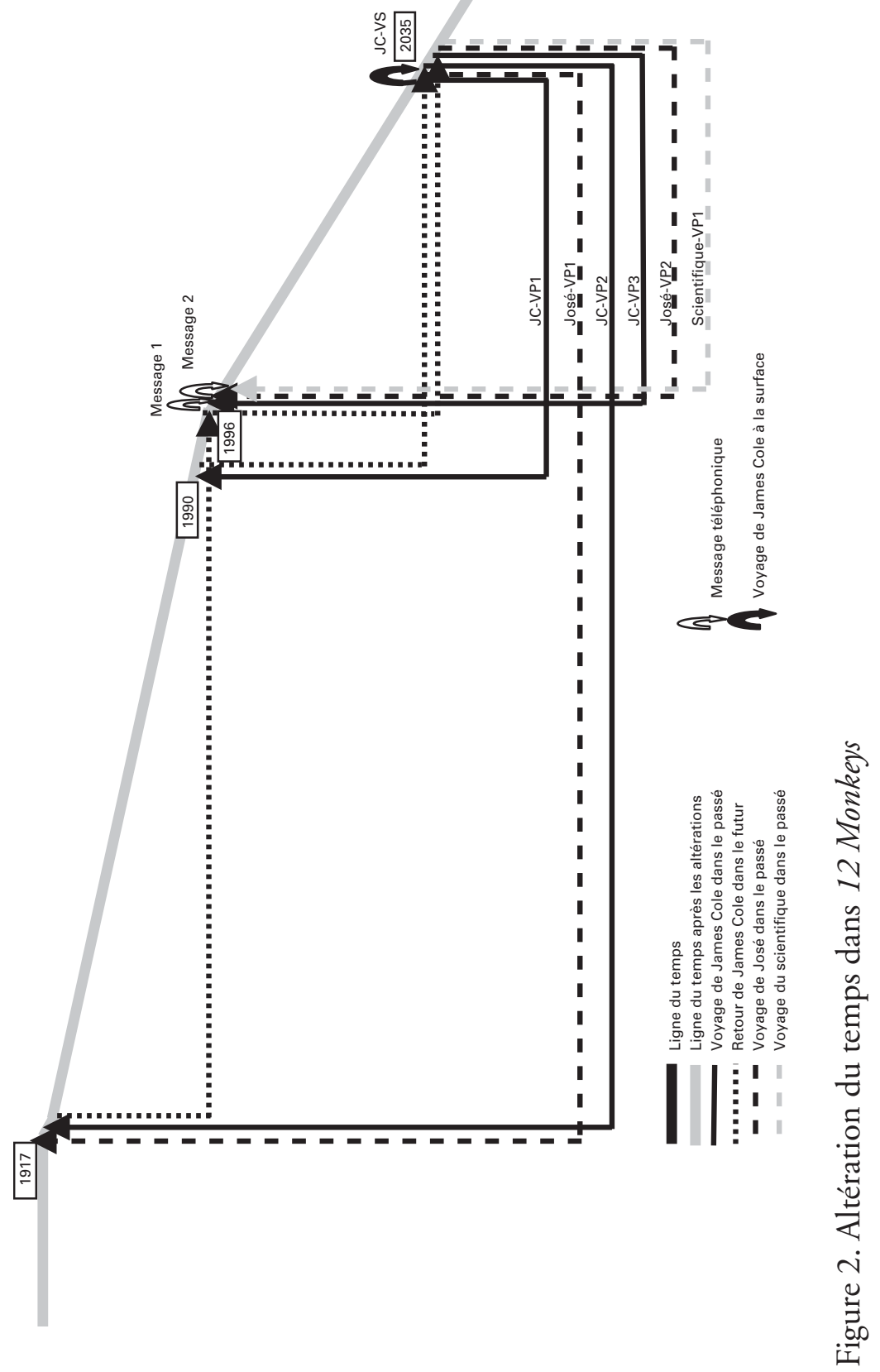

74 CiNéMAS, vol. 13, n³ 
temps, jusqu'à ce qu'il rencontre un univers où il n'y a plus de menace apocalyptique. C'est ce que font les scientifiques du futur. Cole est le bouc émissaire qui altère le passé avec ses voyages et lorsqu'il meurt disparaît avec lui un monstrueux destin alternatif de l'humanité. Seule reste Railly incarnant Cassandre et probablement internée dans un hôpital psychiatrique. Grâce à une adéquate utilisation de la technologie, les scientifiques sont parvenus à ce que toutes les boucles se referment et que nul ne puisse discerner leur intervention dans cette réalité qui n'est autre chose que le résultat de leur manipulation. Passée, présente et future, l'humanité entière est un éternel cobaye dans les mains des savants.

$\mathrm{Au}$ long des trente-trois ans qui séparent la réalisation de $L a$ Jetée et celle de 12 Monkeys, beaucoup de changements ont eu lieu dans le monde. Les cartes géographiques se sont dessinées et redessinées, d'anciens pays ennemis sont devenus amis et vice versa. Diverses révolutions sociales, économiques, culturelles ou politiques ont affecté de vastes secteurs de la population. Les découvertes scientifiques et le développement technologique se sont traduits par d'énormes bouleversements dans nos vies. De nouveaux concepts, notamment ceux du village global et de l'effet de serre, ont vu le jour. Ces transformations ne furent pas toujours positives, mais la chute du mur de Berlin, symbole de la fin de la guerre froide, nous fit croire à l'avènement d'un monde meilleur. Au moins avons-nous osé songer à une diminution du risque d'un conflit nucléaire.

Les métamorphoses survenues dans la société pendant cette période sont clairement illustrées dans la production de Gilliam: la modalité de la fin qu'il propose est marquée par l'accélération et la réitération. En même temps, son film reflète bien l'espace occupé par la femme dans la société postindustrielle. Le rôle des savants, tout en haut de la hiérarchie, renvoie, hélas, à leur ingérence politique, croissante depuis le milieu du siècle passé. Le centre de l'action se trouvant en Amérique et la propagation $\mathrm{du}$ virus atteignant les quatre coins de la planète en disent long sur le nouvel ordre mondial.

D’un point de vue esthétique, la longue durée du film de Gilliam ainsi que la saturation provoquée par les images 
violentes où priment l'accélération, les itérations et les répétitions contrastent avec la capacité évocatrice des vingt-huit minutes des images de Marker, où un narrateur ne glisse qu'un minimum de phrases.

Il serait intéressant d'effectuer d'autres études comparatives de films portant sur la fin du monde afin de vérifier si les variations que nous observons dans l'adaptation de l'œuvre de Marker par Gilliam constituent un phénomène isolé ou si, au contraire, elles obéissent à une tendance qui opérerait plus amplement à la fois dans l'imaginaire contemporain et dans l'expression de celui-ci.

\section{Université du Québec à Montréal}

\section{NOTES}

1. La production de Marker est un court-métrage de 28 minutes et correspond à un photo-montage dans lequel une voix hors champ raconte l'histoire. Par contre, le film de Gilliam correspond au format standard du long-métrage avec une durée de 129 minutes. Dans son mémoire de maîtrise, "Vestiges et vertiges d'une narration rejetée", Manon Martineau (2001) fait une excellente étude comparative de La Jetée, 12 Monkeys et Vertigo, de Hitchcock, où elle aborde, entre autres, ces différences techniques.

2. Possibilité maintenue comme menace au moins pendant toute la guerre froide.

3. Voir son «Discours du palais de Chaillot», 12 septembre 1944, <www.charlesde-gaulle.org>.

4. Voir le site du Musée de l'Homme, <http://www.mnhn.fr/mdh/>.

5. En physique, les particules qui, théoriquement, se déplaceraient à cette vitesse ont été baptisées "tachyons». Nombreux sont les physiciens qui ont étudié les paradoxes de la communication tachyonique, à commencer par Tolman en 1917.

6. De nos jours, nous pourrions même parler avec effroi de l'anticipation que renferme la vision apocalyptique de 12 Monkeys en dressant une comparaison entre celleci et les événements échus au cœur des États-Unis le 11 septembre 2001. Bien que l'organisation d'Al-Qaïda soit loin d'être comparable à l'Armée des douze singes, et que l'attaque du World Trade Center n'ait aucun rapport avec la libération des animaux du Parc zoologique, dans les deux cas nous voyons comment il suffit d'un petit groupe pour répandre la terreur au sein de l'empire américain. Évidemment, la menace que constitue l'anthrax et l'impuissance de la science face à l'irruption du syndrome respiratoire aigu sévère est un écho troublant au virus imaginé par Gilliam en 1995.

7. Voir le site du National Park Service, <http://www.nps.gov/inde/liberty-bell.html>.

8. Curieusement, la première des grandes épidémies aux États-Unis, la fièvre jaune, s'abat sur Philadelphie en 1793 et tue plus de 4000 personnes. Voir <http://www. infoplease.com>.

9. Terry Gilliam aurait copié le travail de Lebbeus Woods, ce qui le conduisit devant les tribunaux. La cour détermina qu'il s'agissait bel et bien d'une reproduction 
non autorisée de la création de Woods dans presque tous ses détails: sol, murs, plafond, sphère, siège. Voir <http://www.copyrightwebsite.com>.

10. À propos de l'histoire de la bombe atomique, voir Castellani et Gigante 1966 et McKay 1984.

11. À propos des épidémies, voir le site de l'Organisation mondiale de la santé, à l'adresse suivante: <http://www.who.in>. Quant à la fonction logistique, voir <http:// www.ukmail.org/ - oswin/logistic. html>.

12. L'image du sauveteur sacrifié évoque immédiatement l'image du Christ. Le film comporte d'autres références bibliques. Par exemple, Jeffrey Goines, chef de l'Armée des douze singes, comme les douze apôtres, déclare: "My father is God" ce qui n'est pas loin de la vérité puisqu'il a créé le virus capable d'éliminer la vie humaine sur la planète. Voir aussi à ce sujet Martineau 2001.

13. Si le docteur Goines se plaint d'elle lorsqu'elle l'appelle, alarmée à cause de la possibilité que Jeffrey ait accès aux virus qu'il étudie, il finit par se rendre à l'évidence qu'elle avait raison lorsqu'il est kidnappé par son propre fils.

14. Remarquons que cet important personnage est féminin, en concordance avec la participation plus active des femmes dans la production de Gilliam que dans celle de Marker.

\section{RÉFÉRENCES BIBLIOGRAPHIQUES}

Castellani et Gigante 1966: Leandro Castellani et Luciano Gigante, Histoire de la bombe atomique. De Démocrite à Oppenheimer, Paris, Laffont, 1966.

Comte-Sponville 1996: André Comte-Sponville, «L'Être-temps. Quelques réflexions sur le temps de la conscience", dans Étienne Klein et Michel Spiro (dir.), Le Temps et sa flèche, Paris, Flammarion, 1996, p. 239-270.

Davies 2002: Paul Davies, "How to Build a Time Machine», Scientific American, septembre 2002, p. 50-55.

Goudot 1999: Marie Goudot, Cassandre, Paris, Autrement, 1999.

Hawking 1989: Stephen W. Hawking, Une brève histoire du temps. Du Big Bang aux trous noirs, Paris, Flammarion, 1989.

Hayles 1990 : N. Katherine Hayles, Chaos Bound. Orderly Disorder in Contemporary Literature and Science, Ithaca, Cornell University Press, 1990.

Marker 1992: Chris Marker, La Jetée, New York, Zone Books, 1992.

Martineau 2001 : Manon Martineau, "Vestiges et vertiges d'une narration re-jetée», Mémoire de maîtrise, Université du Québec à Montréal, 2001.

McKay 1984: Alwyn McKay, The Making of the Atomic Age, Oxford, Oxford University Press, 1984.

Simon 1996: Yves Simon, Le Prochain Amour, Paris, Grasset, 1996.

Watzlawick 1978: Paul Watzlawick, La Réalité de la réalité. Confusion, désinformation, communication, Paris, Seuil, 1978.

Wolf 1985: Christa Wolf, Cassandre. Le récit et les prémisses, Aix-en-Provence, Alinéa, 1985.

Wolf 1999: Christa Wolf, «De Cassandre à Médée», dans Marie Goudot, Cassandre, Paris, Autrement, 1999. 